\title{
Quality of life after endoscopic sinus surgery or balloon sinuplasty: a randomized clinical study*
}

\section{A.J. Bizaki, R. Taulu, J. Numminen, M. Rautiainen}

Department of Otorhinolaryngology, Tampere University and Tampere University Hospital, Tampere, Finland
Rhinology 52: 300-305, 2014

DOl:10.4193/Rhino12.198

*Received for publication:

November 20, 2012

Accepted: February 18, 2014

\begin{abstract}
Objectives: To conduct the first prospective randomized controlled trial that evaluates and compares the clinical outcome and impact of ballonsinuplasty and endoscopic sinus surgery (ESS) on the quality of life of patients suffering from chronic or recurrent rhinosinusitis (CRS) of the maxillary sinus.
\end{abstract}

Methods: Adult patients with symptomatic chronic or recurrent rhinosinusitis without severe findings in the sinuses, as documented in the sinus' Computer Tomography scan and clinical exam, were randomized in 2 groups: ESS and Balloon Sinuplasty. The main variable in our study is the Sinonasal Outcome Test-22 (SNOT 22) and its parameters. These parameters were analysed preoperatively and at 3 months, postoperatively.

Results: There was a subjective improvement in symptoms after surgery. We also noticed an objective improvement in the quality of life of our patients seen as a decrease in the total SNOT 22 score. Both balloon sinuplasty and ESS significantly improved almost all the parameters of SNOT22, with no significant difference being found between these two groups.

Conclusion: Both balloon sinuplasty and endoscopic sinus surgery improved the quality of life of patients with mild chronic or recurrent rhinosinusitis. However, the remarkably higher material cost of balloon sinuplasty compared to ESS sets limits on its broad use. There is an obvious need for further study to find out if, as an office procedure, balloon sinuplasty could deliver cost-savings high enough to cover the higher material cost of balloon sinuplasty. Our study was, however, too small to enable firm conclusions to be drawn.

Key words: balloon sinuplasty, rhino-sinusitis, endoscopic sinus surgery, quality of life, airway inflammation

\section{Introduction}

Rhinosinusitis is a common medical problem with significant symptoms that has a substantial impact on the quality of life $(2,3,10)$. The term rhinosinusitis reflects the concurrent inflammatory and infectious processes that affect the nasal passages and the contiguous paranasal sinuses. Infection, mucosal hyperactivity and anatomical variation all contribute to some extent to the pathophysiology of rhinosinusitis.
Recurrent acute rhinosinusitis is diagnosed when four or more episodes of acute bacterial rhinosinisitis occur per year, without signs or symptoms of rhinosinusitis between episodes ${ }^{(2,3,10)}$. If symptoms last for 12 weeks or longer, in addition to clinical evidence of inflammation or oedema of the middle meatus or ethmoid region, and/or radiographical imaging confirms that paranasal sinus inflammation persists for more than 12 weeks, the patient has chronic rhinosinusitis (CRS) ${ }^{(2,3,10)}$. It is unclear whether recurrent acute rhinosinusitis is actually a separate 
disease category or whether those patients who meet the criteria for recurrent acute rhinosinusitis are simply having frequent exacerbations of chronic rhinosinusitis. For this study, both chronic rhinosinusitis and recurrent acute rhinosinusitis were considered to be one disease.

Chronic rhinosinusitis can be classified as allergic and nonallergic, depending on the presence or not of atopy. In both groups, however, intense eosinophilic infiltration of the mucosa has been noticed. Moreover, the increased levels of immunoglobulin E ( $\mathrm{IgE}$ ) present in allergic CRS has also been reported in CRS even in the absence of a history of allergy and the presence of a negative skin test. However,the basic causative mechanisms responsible for the clinical picture of the disease are not yet clearly definded (2). The symptoms of CRS and recurrent rhinosinusitis vary in severity and prevalence. Nasal obstruction is the most common symptom, followed by facial congestionpressure-fullness, discoloured nasal discharge and hyposmia. An improvement in ventilation and the drainage of the ostiomeatal complex and, at the same time, preservation of the mucosal lining of the upper airways is the main aim of surgical technique development ${ }^{(3)}$.

In this study, we carried out a randomized, clinical study of patients with chronic or recurrent rhinosinusitis of the maxillary sinuses without severe pathology of other sinuses. Our goal was to study the clinical outcome and impact on the quality of life of ballonsinuplasty versus endoscopic sinus surgery (ESS) in patients with chronic or recurrent rhinosinusitis (CRS) of the maxillary sinus.

ESS has become the standard for the surgical treatment of rhinosinusitis. The aim of ESS is to restore the physiological functions of the nasal and paranasal cavities. In many studies, it has been shown that surgery results in an improvement in both subjective and objective findings, in addition to improvements in the quality of life of patients ${ }^{(8,9,11)}$.

In 2002, the balloon sinuplasty technique was introduced in the treatment of ostia of the paranasal sinus system. Balloon sinuplasty is a recently introduced minimally invasive tool in rhinology that uses the concept of remodelling the anatomy of the paranasal sinus ostia without removing mucosal tissue or bone ${ }^{(4)}$. The use of balloon sinuplasty in patients has so far been proven to be feasible and safe ${ }^{(12,13)}$. In a few previous studies, ESS was compared with a hybrid sinus surgical technique where the patients had a combination of ESS and balloon sinuplasty ${ }^{(7)}$. It is, however, also very important to study and compare these two techniques as separate entities. This will not only further evaluate the efficacy of balloon sinuplasty for the treatment of patients with chronic or recurrent rhinosinusitis, but it will also facilitate the identification of those patients that will benefit the most from balloon sinuplasty.

\section{Materials and methods}

Study design

The randomized and controlled clinical study was carried out at the Department of Otolaryngology, Tampere University, Finland. The study comprised 42 patients that were suffering from chronic or recurrent rhinosinusitis. The patients were collected from the outpatient department. To be accepted into the study, all patients needed to qualify for sinus surgery (according to preferred indications for surgical treatment). Informed consent was obtained from all patients in advance.

\section{Inclusion and exclusion criteria}

The following inclusion criteria were used: a) patients had to have been diagnosed with chronic or recurrent rhinosinusitis of the maxillary sinus without severe pathology of other sinuses, b) patients had to be older than 18 years old and younger than 65 years old and c) patients had to fulfil the indications for sinus surgery ${ }^{(3)}$.

In addition to the age limits, the following exclusion criteria were applied during patient recruitment: a) patients with a history of previous sinus operations, b) patients who had been diagnosed with asthma, c) patients with a history of ASAintolerance, d) patients with a history of diabetes or any other systemic disease, e) patients with visible polyps in nasal direct endoscopy and f) patients that were pregnant at the time of enrolment to the study.

\section{Diagnosis}

Routine diagnosis of the underlying pathological condition comprised patient history and direct endoscopic nasal examination. Furthermore, cone beam computed tomography (CBCT) scans of the paranasal sinuses were performed to evaluate their status ${ }^{(1)}$. An experienced radiologist and an otolaryngologist surgeon subsequently interpreted the images.

Patients were allocated into two groups: mild (score per side 1-2) or severe changes (score per side 3-4) at the maxillary sinus and/or the ostiomeatal complex. For classification purposes, the Lund-McKay score of the side with the most severe findings was used. The Lund-McKay score was counted separately for each side and it is a sum of the Lund-McKay score of the maxillary sinus and the ostiomeatal complex. The scale of the Lund-McKay score is from 0 to 2 for each measured area i.e 0 to 2 for the maxillary sinus plus 0 to 2 for the ostiomeatal complex. Therefore, there would be a maximum score of 4 if the maxillary sinus and the ostiomeatal complex were completely blocked and a minimum score of 0 if there were no pathology in the maxillary sinus or the ostiomeatal complex. 
Sino Nasal Outcome Test -22 (SNOT22) Quality of life questionnaire

The SNOT22 questionnaire was used to assess the quality of life of patients. We compared the preoperative SNOT22 score and the SNOT22 score at 3-months, postoperatively. Based on previous validation studies, we considered that the minimally important difference, which is the smallest change in the SNOT22 score that can be detected in a patient, to be 8.9 points ${ }^{(14)}$.

\section{Study groups}

Using MINIM (MS-DOS program for randomization in clinical trials), the patients were randomized into two treatment groups: the endoscopic sinus surgery group and the balloon sinuplasty group. The patients were randomized based on the following variables: a) smoking history, b) age, c) sex and d) Lund McKay score.

Sample size for paired t-test analysis follows approximately the following formula:

$$
n=\frac{\sigma^{2}}{\left(\mu_{1}-\mu_{2}\right)^{2}}[Z(1-\alpha / 2)+Z(1-\beta)]^{2}
$$

where $\sigma$ is estimated standard deviation of the difference, $\mu 1-\mu 2$ is the difference in population means, $Z(1-\alpha / 2)$ and $\mathrm{Z}(1-\beta)$ are values from normal distribution tables for selected alpha and power values. Based on the previously published articles, the highest value for standard deviation has been 1,29. This value was, therefore, selected for the calculations to ensure that not too few patients were selected for the study. Also based on previous studies that used SNOT-22, the clinically significant difference was set to 8.9. With an alpha value of 0.05 and a power value of 0.8 , the calculation gave us approximately 21 study patients for each study group.

\section{Surgical methods}

For both treatment groups, a procedure was performed under regional anesthesia using $250 \mathrm{mg}$ cocaine diluted in $5 \mathrm{ml}$ of $0.1 \mathrm{mg} / \mathrm{ml}$ adrenaline. Additionally, we infused the uncinate process with 4-6 $\mathrm{ml}$ of $10 \mathrm{mg}$ adrenaline cum lidocaine solution. Conscious sedation was achieved for all patients by the intravenous administration of $0.5 \mathrm{ml}$ of $0.5 \mathrm{mg} / \mathrm{ml}$ Rapifen and $0.5 \mathrm{ml}$ of $1 \mathrm{mg} / \mathrm{ml}$ Midazolam.

During surgery, patients in the ESS group underwent the removal of the inferior part of the uncinate process and where necessary the pathology in the ostium was removed to ensure the patency, but the ostium was not enlarged. The principal of balloon sinus dilatation is the cannulation of the sinus ostium with a very thin, flexible guidewire that allows an atraumatic entrance to the sinus, even through a narrowed ostium. Following cannulation and prior to balloon dilatation, it is essential to confirm that the guidewire has entered the sinus. To simplify the process, we used a lighted guidewire called the Luma Sinus Illumination System (Luma light) (Acclarent Inc., Menlo Park, CA, USA). The concept is based on transillumination through the sinus walls for the identification of the guidewire's location. In all the patients, both of the sinuses were treated.

\section{Allergy}

In addition to recording the allergy history of the patients, blood samples were collected and the serum levels of total immunoglobulin E and RAST allergy blood tests were performed on all the patients that participated in our study. More specifically, tests were made for the following allergens common in Finland: a) timothy, b) birch, c) leek, d) dog, e) cat, f) horse, g) mould and h) mite (D. pteronyssinus).

\section{Statistical analyses}

A workstation with SPSS 9.0 software (SPSS Inc., Chicago, IL, USA) was used for the statistical evaluation and graphical representation of the results.

Improvement in quality of life was analysed with paired t-tests. The measurements used in the comparison of the treatments were the individually calculated differences between the preoperational SNOT-22 values and the postoperative 3-month SNOT-22 values. $P$ values smaller than 0.05 were considered to be significant.

\section{Follow-up, and the reporting and assessment of adverse} effects and reactions

There was a systematic follow-up of all the patients and any adverse effects (Figure 1). All patients were evaluated at 3 months postoperatively to determine the effects of the surgical intervention.

\section{Results}

Baseline characteristics / demographics

In total, 46 patients were enrolled on the study. Four female patients dropped out of the treatment programme. Two of them were in the ESS group and two of them in the balloon sinuplasty group. These patients were not included in the statistical analysis because they did not show up for the follow-up control and, therefore, we only have demographics data and the preoperative SNOT22 score for them. They did not give any particular reason for dropping out of the study and there were no complications. They just decided that they did not want to be in the study. The Ethical Committee's decision about our study included a statement that gave patients the right to drop out of the study at any time without giving any reason for their decision. We analysed our data based on the 42 patients ( 13 males and 29 females) that remained in the study. Thus, a total of 42 patients participated in our study, with 21 patients allocated to each treatment group. 


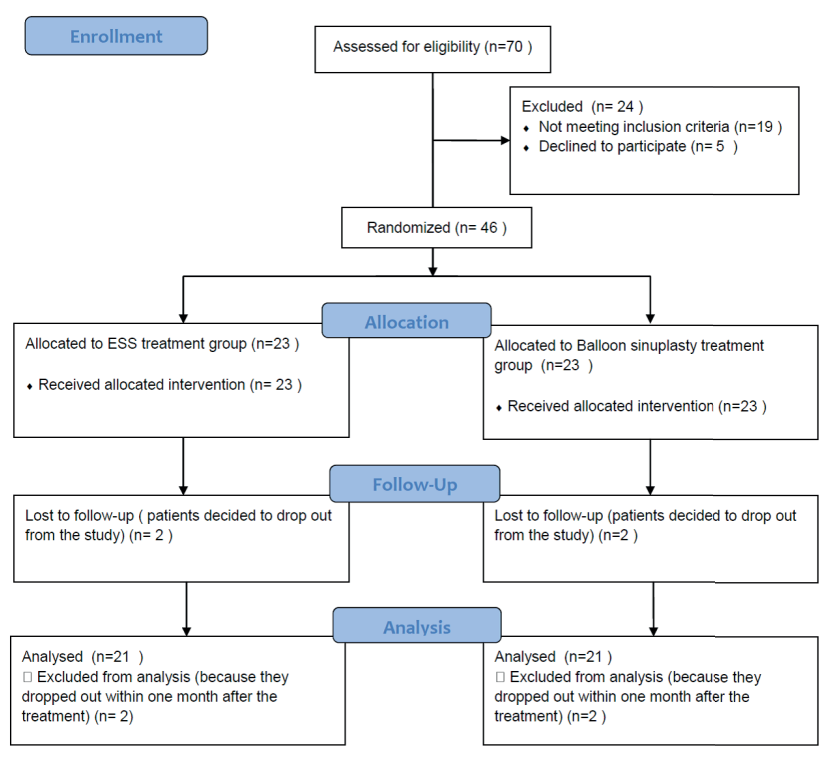

Figure 1. Consort diagram of the study.

Table 1. Demographics.

\begin{tabular}{|ccc|}
\hline & $\begin{array}{c}\text { ESS group } \\
(\mathbf{n}=\mathbf{2 1})\end{array}$ & $\begin{array}{c}\text { Balloon sinus- } \\
\text { plasty (n =21) }\end{array}$ \\
\hline $\begin{array}{c}\text { Mean age of patiens } \\
\pm \text { SEM (years old) }\end{array}$ & $40 \pm 2.6$ & $39.2 \pm 2.3$ \\
\hline Sex & $7 \mathrm{M} ; 14 \mathrm{~F}$ & $9 \mathrm{M} ; 12 \mathrm{~F}$ \\
\hline smoking history & 7 & 6 \\
\hline $\begin{array}{c}\text { usage of nasal steroids } \\
\text { before surgery }\end{array}$ & 14 & 17 \\
\hline $\begin{array}{c}\text { mean duraiton of symptoms } \\
\text { (months) }\end{array}$ & $136.4 \pm 24.9$ & $85.5 \pm 21.1$ \\
\hline $\begin{array}{c}\text { Elevated levels of IgE } \\
\text { (>100 U/ml) }\end{array}$ & 7 & 6 \\
\hline History of allergies & 6 & 9 \\
\hline Positive RAST allergy test & 9 & 9
\end{tabular}

Based on test of homogeneity of variance, the demographic characteristics of patiens were equally distributed between the two treatment groups (no significant difference in variance $p>0.05$ ).

The average age of the patients was about 40 years old. Thirteen patients were smokers and 34 patients had regularly used nasal steroids before the surgery. The mean duration of the symptoms of chronic rhinosinusitis before surgery was $112.4 \pm$ 16.8 months. In 13 patients, the levels of total IgE in serum was elevated (over $100 \mathrm{U} / \mathrm{ml}$ ), and in 18 patients the allergy RAST test was positive (Table 1). The Lund-McKay score was calcula-
Table 2. Difference in SNOT-22 score before and after treatment (mean \pm SEM).

\begin{tabular}{ccccc} 
& $\begin{array}{c}\text { Before } \\
\text { surgery }\end{array}$ & $\begin{array}{c}\text { After } \\
\text { surgery }\end{array}$ & p-value \\
\hline ESS $(\mathrm{n}=21)$ & $46.00 \pm$ & $25.05 \pm$ & $<0.001^{*}$ \\
& 3.27 & 3.24 & \\
\hline Balloon sinusplasty $(\mathrm{n}=21)$ & $43.57 \pm$ & $22.10 \pm$ & $<0.001^{*}$ \\
& 3.64 & 3.28 & \\
& & & & \\
& $p=0.6$ & $p=0.587$ & & \\
& & &
\end{tabular}

* Wilcoxon test showed significant improvement in total SNOT22 score after treatment.

ted based on the findings in the ostiomeatal complex and the maxillary sinuses. Mild disease was found (worst side's score 1-2) in 28 patients and more severe disease (worst side's score 3-4) in 8 patients.

\section{Quality of Life trends}

Statistically significant improvements in quality of life were found between baseline and 3 month follow-up for the total scores of the SNOT22 ( $p<0.001$; Table 1). Cohen's d (effect size) of the difference between baseline and 3-month postop SNOT22 was calculated for both treatment groups and it was significantly high in both treatment groups: Cohen's d was 1.44 for the ESS treatment group and 1.32 for the balloon sinuplasty group. The high difference in effect size in combination to a $p$ value smaller than 0.05 indicates that our sample size was big enough to detect any significant change of total SNOT22 score after treatment. A linear regression analysis was performed to evaluate the percentage of the preoperative total SNOT22 score that affects and predicts the postoperative SNOT22 score. Based on this analysis, in the ESS treatment group, only $20.8 \%$ ( $R$ square $=0.208$ ) of the postoperative total SNOT22 can be predicted from the preoperative total SNOT22 score. This result would suggest that the preoperative total SNOT22 score itself does not have much of an effect on the postoperative SNOT22 score. However, this result could not be statistically verified since the $\mathrm{p}$ value was higher than 0.05 .

When comparing the changes in the total SNOT22 scores of the ESS and balloon sinuplasty groups, we identified no significant differences between the two treatment groups, either preoperatively or 3-months postoperatively (all $p \geq 0.05$; Table 2 ). Cohen's $d$ (effect size) of the difference between the ESS and the balloon sinuplasty group was calculated and it was found to be 0.16 preoperatively and 0.19 at 3-months postoperatively (with $95 \%$ confidence interval). Since $p$ was $>0.05$, we can say that no significant difference was found between the two treatment 


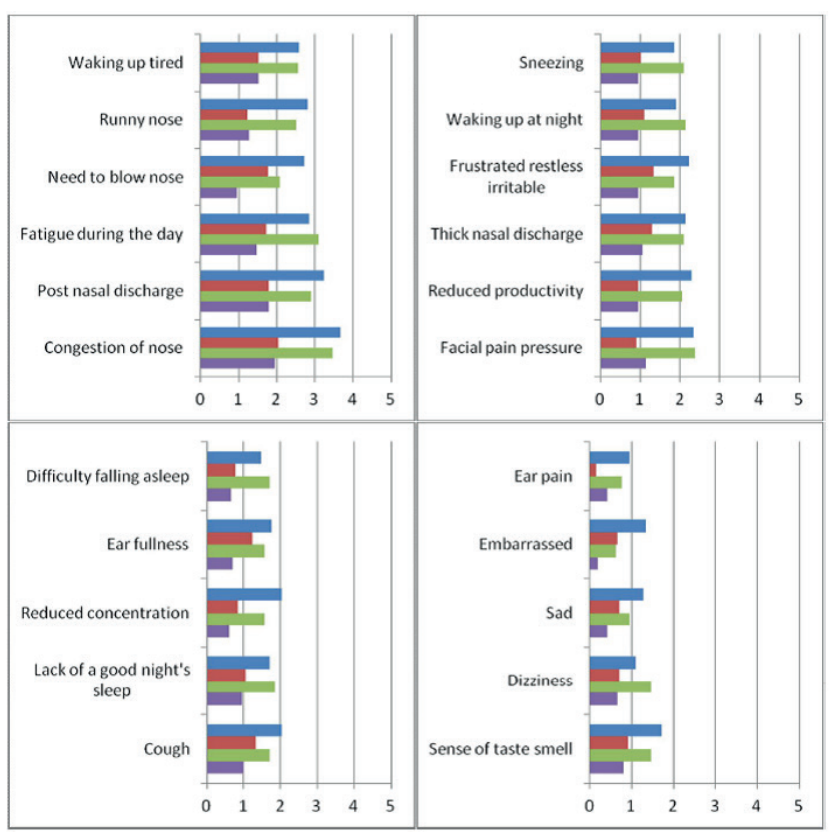

| Before_ESS $\mid$ After_ESS $\mid$ Before_Balloon_Sinuplasty $\|$ After_Balloon_Sinuplasty

Figure 2. Symptoms among the patients with chronic or recurrent rhinosinusitis.

groups. The low effect size of difference indicates, however, that a bigger sample size is required in order to be able to detect any possibly existing significant difference between the two treatment groups.

We cannot be sure whether a difference exist between the two treatment groups and whether or not this is a type II error as our study group was relatively small and the effect size of difference was low. However, based on our current data, we were not able to find any significant difference between the groups. Therefore, more extended studies with a larger number of patients are needed.

The regression to mean has been taken into account. We acknowledged that the phenomenon of "regression to mean" could explain, at least to some degree, the fact that after treatment all the separate variables of SNOT22 were decreased. Neither the sex of patients nor the history of smoking affected the outcome of treatment in any known way $(p>0.05)$. An analysis of the SNOT22 questionnaire's parameters preoperatively showed that nasal congestion, postnasal drip, fatigue, runny nose and facial pain/pressure were the most common symptoms among the patients with chronic or recurrent rhinosinusitis (Figure 2).

\section{Allergy}

A positive RAST allergy test was associated with higher levels of total immunoglobulin $E(p=0.01)$. Neither elevated levels of total immunoglobulin $\mathrm{E}$ (over $100 \mathrm{mg} / \mathrm{dl}$ ) nor a positive RAST allergy test affected the outcome of the treatment $(p>0.05)$.
Table 3. Adverse efffects.

\begin{tabular}{|lccc|}
\hline Adverse effect / complication & $\begin{array}{c}\text { Total of } \\
\text { patients }\end{array}$ & $\begin{array}{c}\text { ESS group } \\
\text { Balloon } \\
\text { group }\end{array}$ \\
\hline Infection & 11 & 4 & 7 \\
\hline Crusting & 5 & 3 & 2 \\
\hline Synechia & 8 & 6 & 2 \\
\hline Anosmia & 5 & 4 & 1 \\
\hline Bleeding & 2 & 1 & 1 \\
\hline
\end{tabular}

With regard to adverse effects, no significant difference was found between ESS and balloon sinusplasty $(p<0.05)$.

\section{Adverse effects}

None of the 42 patients had a major complication. However, minor complications were reported in 21 patients: in 13 patients from the ESS group and in 8 patients from the balloon sinuplasty treatment group. The reported complications in the ESS group were infection (4 patients), crusting (3 patients), synechia (6 patients), anosmia (4 patients) and bleeding (1 patient). In the balloon sinuplasty group, the reported complications were infection (7 patients), crusting (2 patients), synechia (2 patients), anosmia (1 patient) and bleeding (1 patient) (Table 3). Based on Spearman's correlation test, a weak but not statistically significant positive association was found between ESS treatment and the development of synechia $(r=0.243)$ and anosmia $(r=0.221)$ $(p>0.05)$.

\section{Discussion}

Although many studies have examined outcomes after sinus surgery, few have done so in a prospective fashion with randomized groups. There remains a need for prospective trials that compare the methods used in the treatment of chronic sinusitis. In cases of CRS, both balloon sinuplasty and endoscopic sinus treatment seem to improve the quality of life of patients. Three months after treatment, we were not able to find any significant difference between the two methods.

We acknowledge that our study group was small, and that it is likely that there is a difference between these two treatments. However, based on statistical analysis of our present data, we were not able to find any significant difference between these two treatment methods with regard to their effect on the previous studies SNOT22 score. Based on the results of this study, the evidence suggests that chronic rhinosinusitis might be targeted with less invasive treatment methods i.e. balloon sinuplasty in the first instance, with more invasive and radical treatment being reserved for more severe and refractory cases. 
Balloon sinuplasty is a delicate, minimally invasive tool, and our results demonstrate promising outcomes in terms of safety and effectiveness. The results of this study have important implications for future clinical trials designed to evaluate the comparative effectiveness of treatments for CRS.

In our study, patients had sinus disease primarily restricted to the maxillary sinus and ostiomeatal complex with none or minimal changes in other sinuses. In addition, the severity of the findings was relatively mild. It is highly likely that it is difficult to treat certain subgroups of CRS patients, such as those with pansinusitis, with only balloon sinuplasty. In the present study, we did not include any hybrid operations to avoid any confusion in the results caused by combined operative techniques.

This study does not allow conclusions about quality of life (QOL) changes and the efficiency of treatment methods in the long-term. It is probable that certain subgroups of CRS patients, possibly those with severe findings in CT or severe clinical symptoms, will develop recurrent disease over time and a subsequent worsening of QOL measurements. There is a group of patients that suffers from recurrent rhinosinusitis during the infection seasons in winter in spite of proper conservative treatment without any severe findings in CT scans. Some patients have near-normal disease-specific QOL, but have one or more prominent symptoms that still drive them to elect surgical treatment for their disease. This group of patients has little room for improvement in QOL, even though surgery may have been clinically successful.

\section{Conclusion}

Both balloon sinuplasty and endoscopic sinus surgery improved the quality of life of patients with mild, chronic or recurrent rhinosinusitis. However, the remarkably higher material cost of balloon sinuplasty compared to ESS sets limits on its broad use. There is an obvious need for a study to find out if, as an office procedure, balloon sinuplasty could bring cost savings that would cover the higher material cost. To further study balloon sinuplasty's potentials and limits as a method requires more research and long-term studies.

\section{Acknowlegdement}

To research nurse Marja-Leena Oksanen for ensuring patients smooth coordination and support throughout the study.

\section{Authorship contribution}

$A B$ : recruitment of new patients, collection and analysis of data, clinical examination of patients before and after surgery, writing of the manuscript. RT: performed endoscopic sinus surgery and balloon sinuplasty, clinical examination of patients. JN: performed endoscopic sinus surgery and balloon sinuplasty,clinical examination of patients, manuscript review. MR: Principal researcher of the study, recruitment of new patients, performed endoscopic sinus surgery and balloon sinuplasty, manuscript review.

\section{Conflicts of Interest}

None of the authors had any conflict of interest with any financial organization.

\section{References}

1. Ahmad M, Freymiller E.J Cone beam computed tomography: evaluation of maxillofacial pathology.Calif Dent Assoc. 2010; 38: 41-47

2. Desrosiers M, Evans GA, Keith PK, Wright ED, 7. Kaplan A, Bouchard J, et al. Canadian clinical practice guidelines for acute and chronic rhinosinusitis. J Otolaryngol Head Neck Surg. 2011; 40 Suppl 2: S 99-193.

3. Fokkens WJ, Lund VJ, Mullol J, et al. Rhinology Suppl. 2012; (23): 3p preceding table of contents, 1-298.

4. Friedman M, Wilson M. Illumination guided balloon sinuplasty. Laryngoscope. 2009; 119: 1399-1402.

5. Gillett S, Hopkins C, Slack R, Browne JP. A pilot study of the SNOT 22 score in adults with no sinonasal disease. Clin Otolaryngol. 2009: 34: 467-469.

6. Hopkins C, Gillett S, Slack R, Lund VJ, Browne JP. Psychometric validity of the 22-item Sinonasal Outcome Test.Clin Otolaryngol. 2009: 34: 447-454.

7. Kuhn FA, Church CA, Goldberg AN, et al. Balloon catheter sinusotomy: one-year follow-up--outcomes and role in functional endoscopic sinus surgery. Otolaryngo Head Neck Surg. 2008; 139 (Suppl 3): S2737

8. Ling FT,Kountakis SE. Important clinical symptoms in patients undergoing functional endoscopic sinus surgery for chronic rhinosinusitis. Laryngoscope. 2007; 117: 1090-1093.

9. Macdonald KI, MCNally JD, Massoud E Quality of life and impact of surgery on patients with chronic rhinosinusitis. Otolaryngol Head Neck Surg. 2009; 38: 286293.

10. Meltzer EO, Hamilos DL. Rhinosinusitis. diag nosis and management for the clinician: a synopsis of recent consensus guidelines. Mayo Clinic proceedings Mayo Clinic. 2011; 86: 427-443.

11. Poetker DM, Smith TL. Adult chronic rhinosinusitis: surgical outcomes and the role of endoscopic sinus surgery.Curr Opin Otolaryngol Head Neck Surg. 2007; 15: 6-9.

12. Siow JK, Al Kadah B, Werner JA. Balloon sinuplasty: a current hot topic in rhinology. Eur Arch Otorhinolaryngol. 2008; 265: 509-511.
13. Vaughan WC. Review of balloon sinuplasty. Curr Opin Otolaryngol Head Neck Surg. 2008; 16: 2-9.

14. Hopkins C, Gillett S, Slack R, Lund VJ, Browne JP. Psychometric validity of the 22-item Sinonasal Outcome Test. Clin Otolaryngol. 2009; 34: 447-455.

M. Rautiainen

Department of Otorhinolaryngology

Tampere University and Tampere

University Hospital

Tampere

Finland 